\title{
BIOCHEMICAL PROPERTIES OF COFACTOR AND COENZYME METABOLISM IN PORCINE OVIDUCTAL EPITHELIAL CELLS - A MICROARRAY STUDY
}

Ievgeniia Kocherova ${ }^{1}$, Magdalena Kulus $^{2}$, Claudia Dompe ${ }^{3}$, Paweł Antosik², Dorota Bukowska², Bartosz Kempisty ${ }^{1,4,5}$, Paul Mozdziak ${ }^{6}$

\begin{abstract}
The oviduct is a key organ responsible for ultimate oocytes maturation, transport of gametes, sperm capacitation, fertilization, as well as early embryo development. Its innermost layer, oviductal epithelium, represents a highly dynamic structure which undergoes changes in response to different physiological and pathological processes. Previously, the expression profile of genes involved in several important processes in porcine oviductal epithelial cells (OECs) during long-term primary in vitro culture. The present study further characterizes the porcine OECs model using Affymetrix microarray assay and it analyzes gene expression changes observed on the 7th, 15 th and 30th day of culture. 25 genes belonging to "coenzyme metabolic process", "cofactor biosynthetic process" and "cofactor metabolic process" GO BP terms were differentially expressed in culture. The most up-regulated genes were ALDH1L2, P2RX7, PANK1, ACSS2, SCD, AASS and PDK3. In contrast, several genes appeared to be significantly down-regulated, e.g. ACSL4 and HAAO. Considering the biological roles of the most regulated genes, it can be concluded that these changes may indicate the increased metabolic and proliferation activity of studied cells in primary in vitro culture.
\end{abstract}

Running title: Cofactor and coenzyme metabolism in porcine oviductal epithelial cells

Keywords: porcine oviductal epithelial cells, long-term in vitro culture, cofactor metabolism, coenzyme metabolism

\footnotetext{
${ }^{1}$ Department of Anatomy, Poznan University of Medical Sciences, Poznan, Poland

${ }^{2}$ Veterinary Center, Nicolaus Copernicus University in Toruń, 87-100 Torun, Poland

${ }^{3}$ The School of Medicine, Medical Sciences and Nutrition, University of Aberdeen, UK

${ }^{4}$ Department of Histology and Embryology, Poznan University of Medical Sciences, Poznan, Poland

${ }^{5}$ Department of Obstetrics and Gynecology, University Hospital and Masaryk University, Brno, Czech Republic

${ }^{6}$ Physiology Graduate Program, North Carolina State University, Raleigh, North Carolina

* Correspondence: bkempisty@ump.edu.pl

Full list of author information is available at the end of article
} 


\section{Introduction}

The oviduct represents a key organ in the ultimate mutation of oocytes, transport of gametes, sperm capacitation, fertilization, and early embryo development. The mucosal layer of the oviduct can be exposed to various pathogens and endotoxins entering from the peritoneal cavity, follicular fluid, and uterus. Moreover, there is evidence showing that the presence of gametes in the oviduct or follicular granulosa cells, with associated fluid, can modify gene expression of the oviductal epithelial cells. Close to the time of ovulation, the oviduct epithelium is sensitive to local programming stimuli from both male and female gametes, follicular cells, and fluid $[1,2]$. Well characterized in vitro models of the oviduct epithelium are of interest for several general reasons. Firstly, they contribute to the understanding of molecular mechanisms associated with oocyte fertilization and early embryo development; additionally, they provide a tool for reproductive toxicity testing.

Ethical concerns and the lack of healthy tissue sources present limitations for the in vitro model of the human oviductal epithelium [3]. Regarding the anatomical structure and physiological processes, pig reveals some close similarities to human. Porcine derived tissues are readily available from local meat processing facilities, and the procedure for the isolation of epithelial cells is relatively simple and inexpensive. Due to these benefits, pig became one of the favored animal models for the study of the oviductal epithelial cells, it has already been used in studies of reproduction biology [4-6]. The porcine oviductal epithelial cells culture system is easily reproducible, and it represents a long-term culture in the strict sense. Porcine oviductal epithelial cells culture has been reported to be maintained for up to 6 weeks or 10 to 15 passages of cell lines [3].

Affymetrix microarray assays have been previously employed to investigate the expression profiles of genes involved in several important processes in oviductal epithelial cells, such as biological adhesion [7], amino acids metabolism and degradation [8], cell cycle process, proliferation [9], epithelium morphogenesis and oviduct development [10]. The aim of the present study is to further characterize the porcine oviductal epithelial cells model during long-term primary in vitro culture.

\section{Material and Methods Tissue Collection}

The oviducts were collected from 45 crossbred gilts, obtained from a commercial herd. The animals were approximately nine months old and displayed at least two regular oestrous cycles. After reaching the anoestrus phase of the cycle, the animals were slaughtered. Collected oviducts were transported to the laboratory and kept in an isolated container at $38^{\circ} \mathrm{C}$.

\section{Primary Long-Term Cell Culture}

The method used for OEC harvesting and culture has been described in our previous studies $[7,8,11]$. Briefly, collected oviducts were washed twice in Dulbecco's phosphate buffered saline (PBS) and sectioned longitudinally. Then, OECs were gently scratched with surgical blades and subsequently digested for $1 \mathrm{~h}$ at $37{ }^{\circ} \mathrm{C}$ in $1 \mathrm{mg} / \mathrm{mL}$ solution of collagenase I (Sigma Aldrich, St. Louis, MO, USA) and Dulbecco's modified Eagle's medium (DMEM; Sigma Aldrich). The cell suspension was filtered through $40 \mu \mathrm{m}$ pore size strainer and centrifuged for 10 min at $200 \times$ g. After rinsing with PBS, OECs were incubated for another $10 \mathrm{~min}$ at $37^{\circ} \mathrm{C}$ with $0.5 \%$ Trypsin/EDTA (Sigma Aldrich), filtered and centrifuged as described above, resuspended in supplemented DMEM (10\% FCS, $100 \mathrm{U} / \mathrm{mL}$ penicillin, 100 $\mu \mathrm{g} / \mathrm{mL}$ streptomycin, and $1 \mu \mathrm{g} / \mathrm{mL}$ amphotericin B) and seeded onto culture dishes. The cells were cultured for up to 30 days $\left(37^{\circ} \mathrm{C}, 5 \% \mathrm{CO}_{2}\right)$ and the medium of the cultures was changed every three days. Once OECs reached confluence of around 75-80\%, they were digested with $0.025 \%$ Trypsin/EDTA (Cascade Biologics, Portland, USA), centrifuged and passaged to another culture dish at a seeding density of $2 \times 10^{4}$ cells $/ \mathrm{cm}^{2}$.

\section{RNA Isolation from Oviductal Epithelial Cells}

OECs were suspended in TRI Reagent (Sigma Aldrich) after $24 \mathrm{~h}$ and 7, 15, and 30 days of culture. The total mRNA isolation was performed using RNeasy MinElute cleanup Kit (Qiagen, Hilden, Germany). The quantity of isolated mRNA was assessed by measuring the absorbance at a wavelength of $260 \mathrm{~nm}$, whereas the purity of RNA samples was determined as $260 \mathrm{~nm} / 280 \mathrm{~nm}$ ratio of absorbance using a spectrophotometer (Bioanalyzer 2100, Agilent Technologies, Inc., Santa Clara, CA, USA). Each mRNA sample was diluted to $100 \mathrm{ng} / \mu \mathrm{L}$ concentration.

\section{Microarray expression analysis and statistics}

Total RNA (100 ng) from each pooled sample was subjected to two rounds of sense cDNA amplification (Ambion® WT Expression Kit). The obtained cDNA was used for biotin labeling and fragmentation using Affymetrix GeneChip $®$ WT Terminal Labeling and Hybridization (Affymetrix, Santa Clara, CA, USA). Biotin-labeled fragments of cDNA $(5.5 \mu \mathrm{g})$ were hybridized to the Affymetrix® Porcine Gene 1.1 ST Array Strip $\left(48^{\circ} \mathrm{C} / 20 \mathrm{~h}\right)$. Microarrays were then washed and stained according to the technical protocol, using the Affymetrix GeneAtlas Fluidics Station. The array strips were scanned employing the Imaging Station of the GeneAtlas System. Preliminary analysis of the scanned chips was performed using the Affymetrix GeneAtlas $^{\mathrm{TM}}$. Operating Software. The quality of gene expression data was confirmed according to the 
quality control criteria provided by the software. The obtained CEL files were imported into downstream data analysis software.

All of the presented analyses and graphs were generated using Bioconductor and R programming languages. Each CEL file was merged with a description file. To correct background, normalize, and summarize the results, we used the Robust Multiarray Averaging (RMA) algorithm. To determine the statistical significance of the analyzed genes, moderated t-statistics from the empirical Bayes method were performed. The obtained $\mathrm{p}$-value was corrected for multiple comparisons using Benjamini and Hochberg's false discovery rate. The selection of significantly altered genes was based on a p-value smaller than 0.05 and expression higher than two-fold.

Differentially expressed genes were subjected to selection through examination of genes involved in cell migration regulation. The differentially expressed gene list (separated for up- and down-regulated genes) was uploaded on the DAVID software (Database for Annotation, Visualization and Integrated Discovery) [12], where genes belonging to the terms of all three Gene Ontology (GO) domains were extracted. Expression data of these genes were also subjected to the hierarchical clusterization procedure, with their expression values presented as a heatmap.

Subsequently, we analyzed the relation between the genes belonging to chosen GO terms with the GOplot package [13]. The GoPlot package calculated the z-score: the number of up- regulated genes minus the number of down- regulated genes divided by the square root of the count. This information allowed to estimate the change course of each gene-ontology term.

Interactions between differentially expressed genes/proteins belonging to the studied gene on- tology group were investigated using the STRING10 software (Search Tool for the Retrieval of Interacting Genes) [14]. The list of the names of the genes was used as a query for interaction prediction. The search criteria were based on co-occurrences of genes/proteins in scientific texts (text mining), co-expression, and experimentally observed interactions. The results of such analyses generated a gene/protein interaction network where the intensity of the edges reflected the strength of the interaction score.

Finally, the functional interaction between genes that belong to the chosen GO BP terms were investigated with the REACTOME FIViz application to the Cytoscape 3.7.2 software. The ReactomeFIViz app is designed to find pathways and network patterns related to cancer and other types of diseases. This application accesses the pathways stored in the Reactome database, allowing to perform pathway enrichment analysis for a set of genes, to visualize hit pathways using manually laid-out pathway diagrams directly in Cytoscape, and to investigate functional relationships among genes in hit pathways. The application can also access the Reactome Functional Interaction (FI) network, a highly reliable and manually curated pathway-based protein functional interaction network covering over $60 \%$ of human proteins.

\section{Ethical approval}

The research related to animal use has been complied with all the relevant national regulations and instructional policies for the care and use of animals. Bioethical Committee approval no. 246/1992.

\section{Results}

Whole transcriptome profiling with Affymetrix microarrays allowed analysis of the gene expression changes between 7,15 , and 30 days of porcine oviductal epithelial cell culture. Using Affymetrix $®$ coenzyme metabolic process

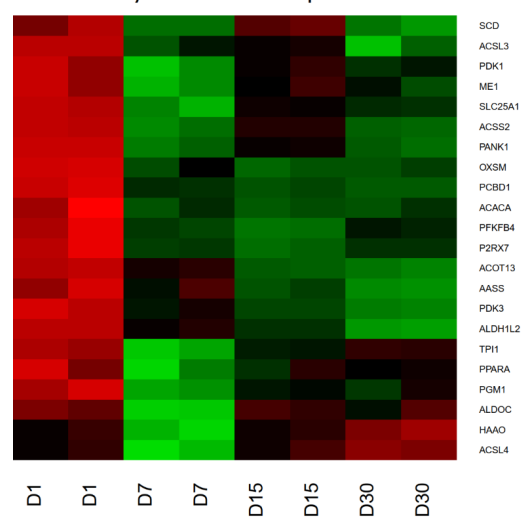

cofactor biosynthetic process

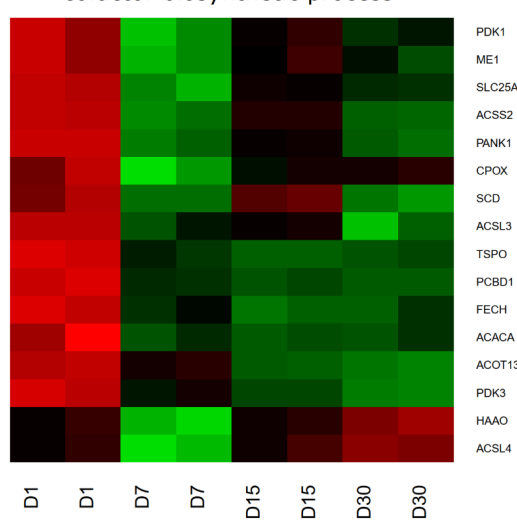

cofactor metabolic process

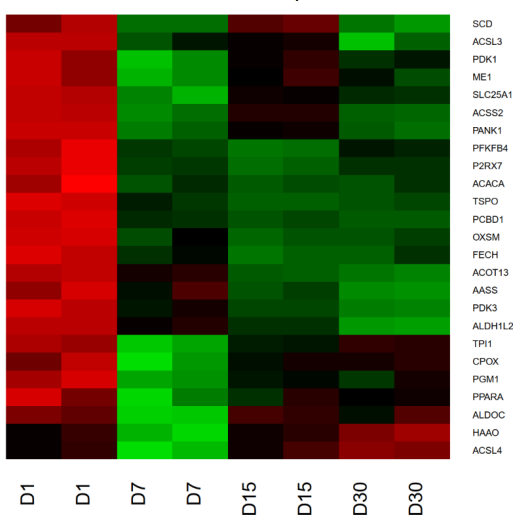

FIGURE 1 Heat map representation of differentially expressed genes belonging to the to "coenzyme metabolic process", "cofactor biosynthetic process" and "cofactor metabolic process" GO BP terms. Arbitrary signal intensity acquired from microarray analysis is represented by colours (green, higher; red, lower expression). Log2 signal intensity values for any single gene were resized to Row Z-Score scale (from -2 , the lowest expression to +2 , the highest expression for single gene) 
TABLE 1 Gene symbols, fold change in expression ratio, Entrez gene IDs, corrected p values and mean value of fold change ratio of studied genes

\begin{tabular}{|c|c|c|c|c|c|c|c|c|}
\hline $\begin{array}{c}\text { GENE } \\
\text { SYMBOL }\end{array}$ & $\begin{array}{l}\text { RATI0 } \\
\text { D7/D1 }\end{array}$ & $\begin{array}{c}\text { RATIO } \\
\text { D15/D1 }\end{array}$ & $\begin{array}{c}\text { RATIO } \\
\text { D30/D1 }\end{array}$ & $\begin{array}{c}\text { ADJUSTED } \\
\text { P VALUE } \\
\text { D7/D1 } \\
\end{array}$ & \begin{tabular}{|c|} 
ADJUSTED \\
P VALUE \\
D15/D1 \\
\end{tabular} & \begin{tabular}{|c|} 
ADJUSTED \\
P VALUE \\
D30/D1 \\
\end{tabular} & $\begin{array}{l}\text { ENTREZ } \\
\text { GENE ID }\end{array}$ & $\begin{array}{l}\text { MEAN } \\
\text { RATIO }\end{array}$ \\
\hline ACSL4 & 2,078475 & $-1,049840$ & $-1,389865$ & 0,000888 & 0,778847 & 0,027679 & 448980 & $-0,120410$ \\
\hline$H A A O$ & 2,260462 & 1,012229 & $-1,484548$ & 0,000495 & 0,948252 & 0,010911 & 100514283 & 0,596048 \\
\hline PPARA & 2,156741 & 1,463697 & 1,431760 & 0,001697 & 0,036412 & 0,040484 & 397239 & 1,684066 \\
\hline CPOX & 2,562460 & 1,513475 & 1,395239 & 0,000490 & 0,019946 & 0,042725 & 100511474 & 1,823725 \\
\hline$A L D O C$ & 3,100575 & 1,215495 & 1,317360 & 0,000301 & 0,274540 & 0,110445 & 100512013 & 1,877810 \\
\hline PGM1 & 2,424519 & 1,680756 & 1,691567 & 0,000276 & 0,002924 & 0,002153 & 397566 & 1,932281 \\
\hline TPI1 & 2,751177 & 1,737387 & 1,421065 & 0,000059 & 0,000779 & 0,006133 & 100157582 & 1,969876 \\
\hline$M E 1$ & 2,691975 & 1,528067 & 1,911722 & 0,000358 & 0,017206 & 0,001723 & 397538 & 2,043921 \\
\hline ACSL3 & 2,049555 & 1,676912 & 2,729973 & 0,002097 & 0,008605 & 0,000218 & 100233169 & 2,152147 \\
\hline FECH & 2,109918 & 2,722032 & 2,420194 & 0,000678 & 0,000117 & 0,000152 & 100322873 & 2,417382 \\
\hline SLC25A1 & 3,272247 & 1,857686 & 2,239022 & 0,000043 & 0,000649 & 0,000117 & 100154310 & 2,456318 \\
\hline OXSM & 2,234415 & 2,697009 & 2,514316 & 0,000560 & 0,000143 & 0,000144 & --- & 2,481914 \\
\hline PFKFB 4 & 2,978245 & 3,699473 & 2,573760 & 0,000132 & 0,000034 & 0,000122 & 100158056 & 3,083826 \\
\hline$P D K 1$ & 4,856476 & 1,981458 & 2,658224 & 0,000100 & 0,004418 & 0,000537 & 100286871 & 3,165386 \\
\hline PCBD1 & 2,831266 & 3,234674 & 3,436204 & 0,000047 & 0,000015 & 0,000007 & 100155605 & 3,167382 \\
\hline$A C A C A$ & 3,411749 & 3,730045 & 3,501388 & 0,000588 & 0,000307 & 0,000279 & 397324 & 3,547727 \\
\hline TSPO & 3,123906 & 3,962945 & 3,669010 & 0,000048 & 0,000012 & 0,000009 & 396592 & 3,585287 \\
\hline ACOT13 & 2,203769 & 4,264528 & 4,968930 & 0,000233 & 0,000008 & 0,000003 & 100514587 & 3,812409 \\
\hline$S C D$ & 5,037882 & 1,402767 & 5,796847 & 0,000092 & 0,087483 & 0,000025 & 396670 & 4,079166 \\
\hline PDK3 & 2,773770 & 4,066151 & 5,486628 & 0,000182 & 0,000024 & 0,000006 & 100153858 & 4,108850 \\
\hline AASS & 2,332931 & 4,119242 & 6,132447 & 0,007329 & 0,000476 & 0,000092 & 100513962 & 4,194873 \\
\hline ACSS2 & 6,349718 & 2,503393 & 5,620930 & 0,000007 & 0,000095 & 0,000002 & 100153866 & 4,824680 \\
\hline PANK1 & 7,855562 & 3,491674 & 7,158650 & 0,000007 & 0,000033 & 0,000002 & 100154650 & 6,168629 \\
\hline$P 2 R X 7$ & 6,165142 & 8,522042 & 5,795398 & 0,000027 & 0,000007 & 0,000010 & 497623 & 6,827528 \\
\hline$A L D H 1 L 2$ & 4,535793 & 8,626347 & 22,102755 & 0,000038 & 0,000004 & 0,000001 & 100151976 & 11,754965 \\
\hline
\end{tabular}

D7/D1
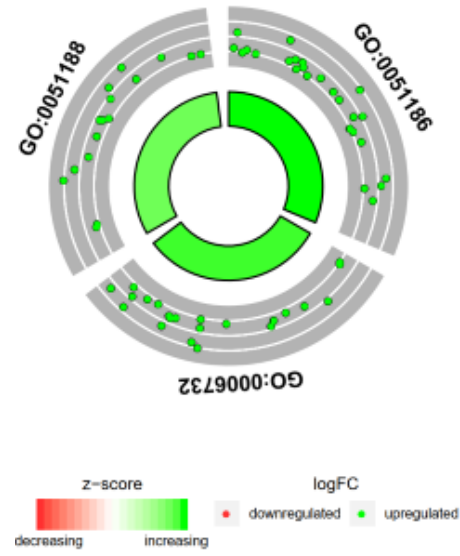

D15/D1

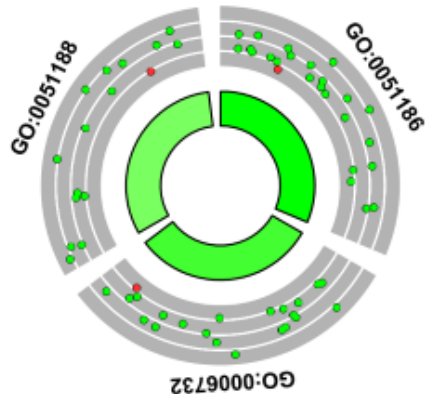

¿\&L9000:0९
D30/D1
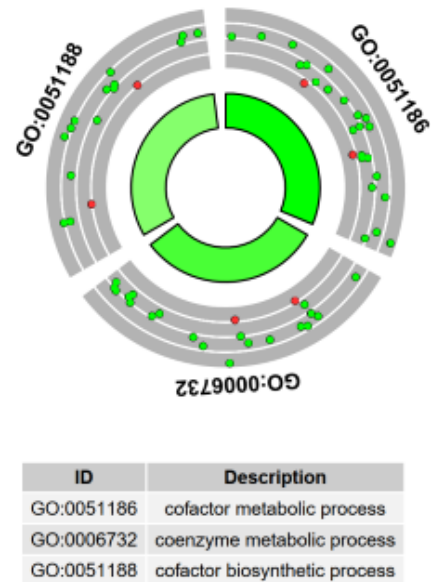

FIGURE 2 The circle plot showing the differently expressed genes and z-score of the "coenzyme metabolic process", "cofactor biosynthetic process" and "cofactor metabolic process" GO BP terms. The outer circle shows a scatter plot for each term of the fold change of the assigned genes. Green circles display up- regulation and red ones down- regulation. The inner circle shows the z-score of each GO BP term. The width of the each bar corresponds to the number of genes within GO BP term and the color corresponds to the z-score 


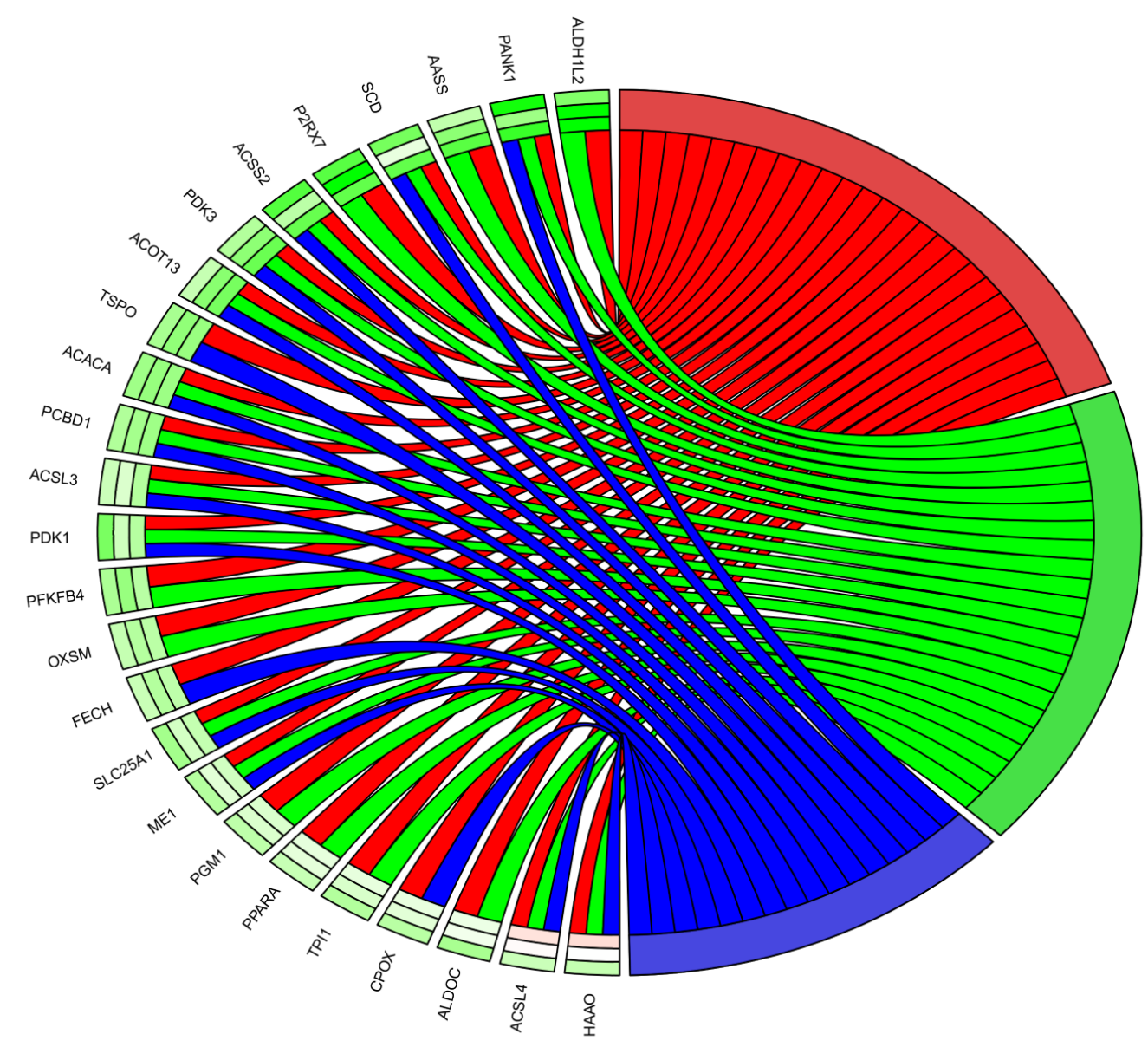

GO Terms

cofactor metabolic process

coenzyme metabolic process

cofactor biosynthetic process

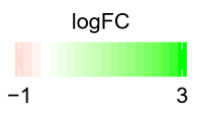

FIGURE 3 The representation of the mutual relationship of differently expressed genes that belong to "coenzyme metabolic process", "cofactor biosynthetic process" and "cofactor metabolic process" GO BP terms. The ribbons indicate which gene belongs to which categories. The middle circle represents logarithm from fold change (LogFC) between D7/D1, D15/D1 and D30/D1 respectively. The color of each block corresponds to the LogFC of each gene (green - up-regulated, red - down-regulated). The genes were sorted by logFC from most to least changed gene

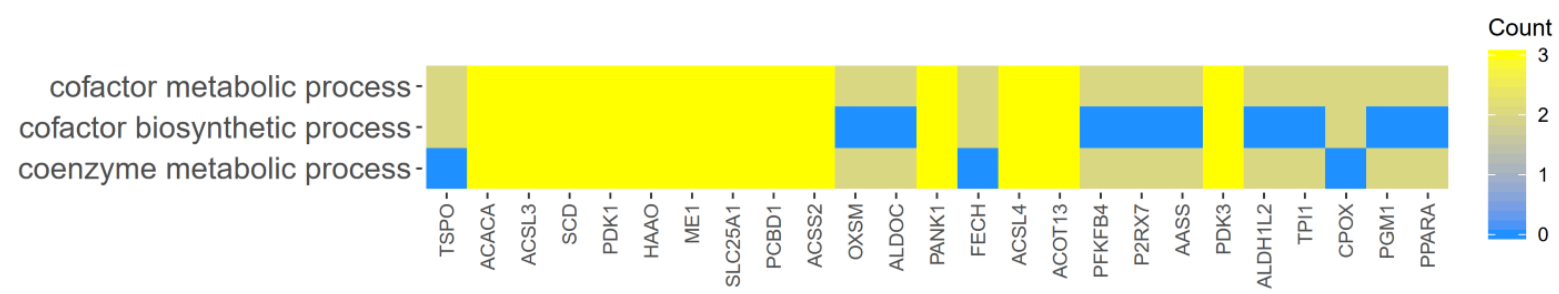

FIGURE 4 Heatmap showing the gene occurrence between chosen differently expressed genes that belongs to "coenzyme metabolic process", "cofactor biosynthetic process" and "cofactor metabolic process" GO BP terms. The yellow color is associated with gene occurrence in the GO Term. The intensity of the color is corresponding to amount of GO BP terms that each gene belongs to

Porcine Gene 1.1 ST Array Strip, the expression of 12257 transcripts was examined and genes were considered as differentially expressed when presented a fold change higher than abs (2) and corrected p-values lower than 0.05 . This set of genes consisted of 2533 different transcripts.
DAVID (Database for Annotation, Visualization and Integrated Discovery) software was used for the extraction of gene ontology biological process terms (GO BP) that contained differentially expressed transcripts. Up- and down-regulated gene sets were subjected to DAVID searches separately, 


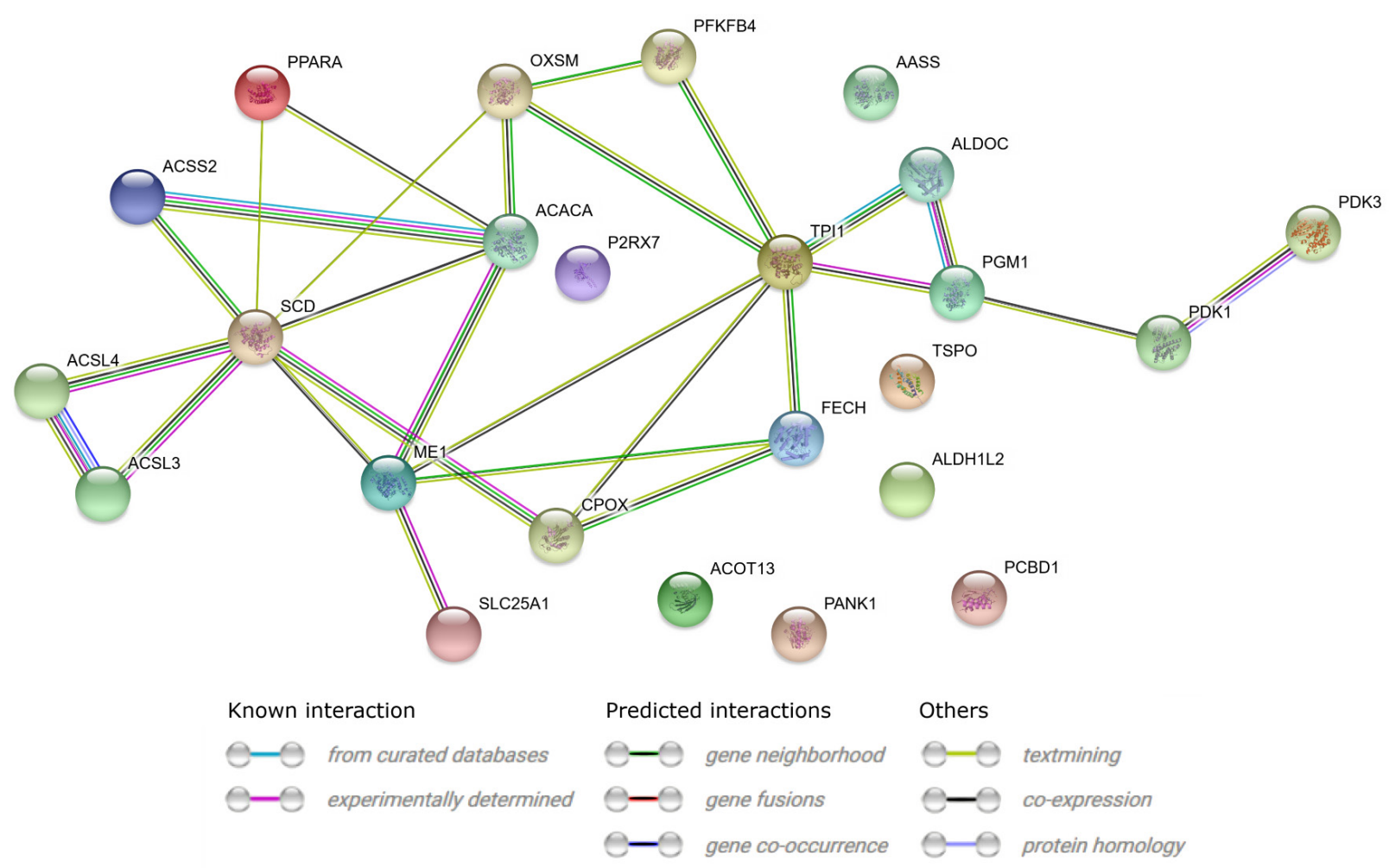

FIGURE 5 STRING-generated interaction occurrence between chosen differently expressed genes that belongs to the to "coenzyme metabolic process", "cofactor biosynthetic process" and "cofactor metabolic process" GO BP terms. The intensity of the edges reflects the strength of interaction score
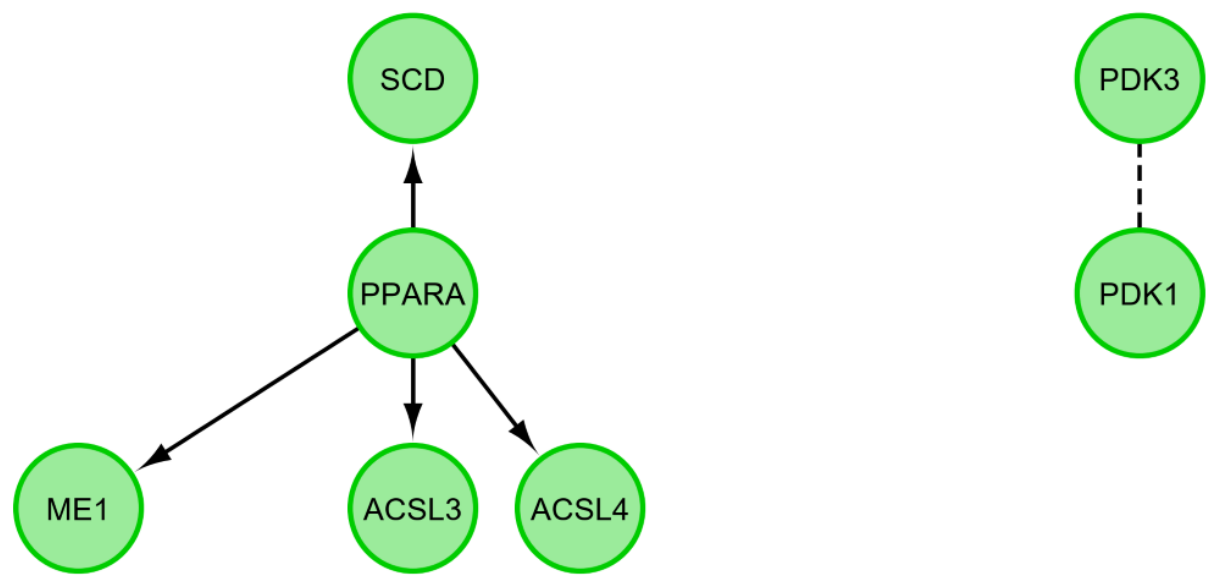

FIGURE 6 Functional interaction (FI) between chosen differently expressed genes that belongs to the to "coenzyme metabolic process", "cofactor biosynthetic process" and "cofactor metabolic process" GO BP terms. In following figure "->" stands for activating/catalyzing, "-|" for inhibition, "-" for FIs extracted from complexes or inputs, and “---" for predicted FIs

performed only on selected gene sets with adjusted p-value lower than 0.05. The DAVID software analysis showed that the differently expressed genes belonged to 657 Gene ontology terms 25 genes that belong to "coenzyme metabolic process", "cofactor biosynthetic process" and "cofactor metabolic process" GO BP terms were chosen for further analysis. These sets of genes were subjected to hierarchical clusterization procedure and presented as heatmaps (Fig. 1). Gene symbols, fold changes in ex- pression, Entrez gene IDs and corrected p values of the genes were shown in table 1.

The enrichment of each GO BP term was calculated as z-score and shown on the circle diagram (Fig. 2).

Chosen GO BP terms contained 166 differently expressed genes. Therefore, the calculated mean value of the mean fold change ratio of each gene between 7, 15 and 30 days of culture. Based on that criteria, 10 most downregulated and 10 most up-regulated genes were chosen for further analysis. 
In Gene Ontology database genes that formed one particular GO group can also belong to other GO term categories. Accordingly, we explored the gene intersections between selected GO BP terms. The relation between those GO BP terms was presented as a circle plot (Fig. 3) as well as a heatmap (Fig. 4).

The STRING-generated interaction network was created among differentially expressed genes belonging to each selected GO BP terms. Using such prediction method, we obtained a molecular interaction network formed between the proteins derived from the genes of interest (Fig. 5). Finally, we investigated the functional interactions of the chosen genes with REACTOME FIViz app to Cytoscape 3.7.1 software. The results were shown in Fig. 6.

\section{Discussion}

Whole transcriptome profiling approach was employed to analyse the genes belonging to "coenzyme metabolic process", "cofactor biosynthetic process" and "cofactor metabolic process" GO BP terms. In general, the chosen ontology groups are associated with chemical reactions and cell signalling pathways resulting in the formation of cofactors, as well as with reactions and pathways that involve coenzymes and cofactors.

The most up-regulated gene was $A L D H 1 L 2$ which encodes a mitochondrial isoform of a similar cytosolic enzyme, ALDH1L1 possessing 10-formyltetrahydrofolate dehydrogenase/hydrolase activities [15]. While the proposed function of the cytosolic enzyme is the overall regulation of the flux of one-carbon groups through folate pool, its mitochondrial counterpart ALDH1L2 may be also involved in producing formate, a pathway shuttling one-carbon groups from mitochondria to cytosol. Additionally, this enzyme might be involved in the regulation of mitochondrial protein biosynthesis [16]. The reaction catalyzed by ALDH1L irreversibly removes carbon groups from the folate pool completing the folate-dependent biosynthetic pathways, which might fulfill several metabolic functions including clearance of excess of folate-bound one-carbon groups [15]. It should be mentioned that, in contrast to cytosolic ALDH1L1, mitochondrial ALDH1L2 is present at detectable levels in several cancer cell lines, denoting different mechanisms of regulation of these enzymes [15].

The next up-regulated gene was $P 2 R X 7$ which generates a plasma membrane receptor for extracellular ATP. P2RX7 is expressed at a high level by immune and tumor cells; however recent studies have shown its presence in an increasing number of different cells lineages, including stem, neural, bone, endothelial, muscle, and skin cells [17]. P2RX7 was revealed in the oviductal epthelium, and is a cation-selective plasma membrane channel that undergoes, when over-activated, a channel-to-pore transition responsible for a reversible permeabili- zation of the plasma membrane to low molecular weight aqueous solutes [18]. P2RX7 induces various downstream cell cascades, including inflammatory molecule release, cell proliferation, apoptosis, metabolic events, and phagocytosis [17]. Previously, P2RX7 has been mostly associated with cytotoxicity and proinflammatory cytokines release. However, recent studies revealed that P2RX7 has a potent growth-promoting activity and has been implicated in tumor progression [18].

The upregulation of genes related to coenzyme A (CoA) metabolism, i.e. PANK1 and ACSS2. PANK1 encodes a member of the pantothenate kinase family, which catalyzes the cytosolic phosphorylation of pantothenate (vitamin B5), N-pantothenoylcysteine and pantetheine, and it controls the overall rate of CoA biosynthesis was revealed by the microarray analysis [19]. CoA plays a central role in the metabolism of carboxylic acids, including short- and longchain fatty acids $[20,21]$. Fatty acids are incorporated into membranes and signaling molecules, and they participate in energy metabolism. These essential functions require activation of fatty acids by acyl-CoA synthetases, e.g. ACSS2, forming an activating thioester linkage between the fatty acid and CoA [22]. The same as PANK1, ACSS2 gene was up-regulated at D7 and D30 during long-term OECs culture.

Additionally, up-regulation of stearoyl-CoA desaturase gene $(S C D)$, which product is an endoplasmic reticulum enzyme that catalyzes the biosynthesis of monounsaturated fatty acids from saturated fatty acids [23] was observed in the array analysis. SCD plays an important role in linking lipid metabolism with adaptive stress signaling and in disorders such as metabolic syndrome, cardiovascular disease and cancer [24].

The next highly up-regulated gene was AASS, which encodes alpha-aminoadipic semialdehyde synthase, a bifunctional protein that catalyzes the first 2 steps in the lysine degradation pathway [25]. The role of AASS in lysine metabolism has been confirmed by earlier studies, where the inactivating mutations in the AASS gene were shown to be a cause of hyperlysinemia [26].

The similar pattern of expression was observed in the case of $P D K 3$ gene. Pyruvate dehydrogenase kinases (PDKs) characterize the pyruvate dehydrogenase complex (PDC) phosphatase inhibitors, which enhances cell glycolysis and facilitates tumor cell proliferation $[27,28]$. Pyruvate dehydrogenase kinase 3 (PDK3) is a mitochondrial protein considered as a potential pharmacological target for different types of cancer [29].

The previously mentioned genes encode proteins participating in metabolism activation and cell proliferation. During long-term OECs culture, the up-regulation of these genes may be due to the processes related to cell expansion following the initial seeding on culture vessels. 
There are several genes belonging to the chosen GO BP terms which were significantly down-regulated after 15 days of in vitro culture. The most down-regulated gene was ACSL4, which plays an essential regulatory role in the ferroptotic cell death process. Acsl4 is responsible for shaping the cellular lipidome and its expression has been reported to correlate with sensitivity to this form of necrotic cell death [30]. Knockdown of ACSL4 has been shown to inhibit erastin-induced ferroptosis in HepG2 and HL60 cell lines, whereas overexpression of ACSL4 by gene transfection restored sensitivity of LNCaP and K562 cells to erastin [31]. When compared to D1 and D7, the down-regulation of ACSL4 in OECs after 15 days in culture may indicate lower sensitivity to ferroptotic necrosis.

The next gene that was down-regulated during the culture period is $H A A O$. Known as 3-hydroxyanthranilate-3,4-dioxygenase, the product of this gene is an enzyme that catalyzes the biosynthetic pathway from tryptophan to quinolonate. HAAO hypermethylation, which leads to the repression of gene transcription, appears to be a frequent event in endometrial carcinomas [32,33]. Given this, $H A A O$ down-regulation may be related to OECs proliferation in culture.

In conclusion, the current results suggest differential expression of genes belonging to "coenzyme metabolic process", "cofactor biosynthetic process" and "cofactor metabolic process" GO BP terms in porcine OECs during 30 days of culturing. Considering the biological roles of the most up- and down-regulated genes, it can be concluded that these changes may indicate the increased metabolic and proliferation activity of studied cells in primary in vitro culture. This assumption is consistent with our earlier studies showing that, after long-term culture, the OECs still proliferate and maintain their tube forming properties $[10,34]$.

\section{Corresponding author}

Bartosz Kempisty, Department of Anatomy, Poznan University of Medical Sciences, 6 Święcickiego St., 60-781 Poznań, Poland, Tel./ Fax: +48 61 8546565, e-mail: bkempisty@ump.edu.pl.

\section{Conflict of interest statement}

The authors declare they have no conflict of interest.

\section{References}

1. Hunter RHF. Components of oviduct physiology in eutherian mammals Biol Rev. 2012;87:244-55; DOI:10.1111/j.1469-185X.2011.00196.x.

2. Fernandez-Fuertes B, Rodríguez-Alonso B, Sánchez JM, Simintiras CA, Lonergan P, Rizos D, Fernandez-Fuertes B, Rodríguez-Alonso B, Sánchez JM, Simintiras CA, Lonergan P, Rizos D. Looking at the big picture: understanding how the oviduct $\mathrm{s}$ dialogue with gametes and the embryo shapes reproductive success. Anim Reprod. 2018;15:751-64; DOI:10.21451/1984-3143-AR2018-0036.

3. Chen S, Einspanier R, Schoen J. Long-term culture of primary porcine oviduct epithelial cells: Validation of a comprehensive in vitro model for reproductive science. Theriogenology. 2013;80:862-9; DOI:10.1016/j. theriogenology.2013.07.011.

4. D'cruz OJ, Erbeck D, Uckun FM. A Study of the Potential of the Pig as a Model for the Vaginal Irritancy of Benzalkonium Chloride in Comparison to the Nonirritant Microbicide PHI-443 and the Spermicide Vanadocene Dithiocarbamate. Toxicol Pathol. 2005;33:465-76; DOI:10.1080/01926230590959866.
5. Mueller A, Siemer J, Renner S, Hoffmann I, Maltaris T, Binder H, Beckmann Mw, Dittrich R. Perfused Non-Pregnant Swine Uteri: A Model for Evaluating Transport Mechanisms to the Side Bearing the Dominant Follicle in Humans. J Reprod Dev. 2006;52:617-24; DOI:10.1262/ jrd.18021.

6. Vodička $\mathrm{P}$, Smetana $\mathrm{K}$, Dvořánková $\mathrm{B}$, Emerick $\mathrm{T}, \mathrm{Xu}$ Yz, Ourednik J, Ourednik V, Motlík J. The Miniature Pig as an Animal Model in Biomedical Research. Ann N Y Acad Sci. 2005;1049:161-71; DOI:10.1196/ annals.1334.015.

7. Budna-Tukan J, Światły-Błaszkiewicz A, Celichowski P, Kałużna S, Konwerska A, Sujka-Kordowska P, Jankowski M, Kulus M, Jeseta M, Piotrowska-Kempisty H, Józkowiak M, Antosik P, Bukowska D, Skowroński MT, Matysiak J, Nowicki M, Kempisty B. "Biological Adhesion" is a Significantly Regulated Molecular Process during Long-Term Primary In Vitro Culture of Oviductal Epithelial Cells (Oecs): A Transcriptomic and Proteomic Study. Int J Mol Sci. 2019;20:3387; DOI:10.3390/ijms20143387.

8. Kranc W, Jankowski M, Budna J, Celichowski P, Khozmi R, Bryja A, Borys S, Dyszkiewicz-Konwińska M, Jeseta M, Magas M, Bukowska D, Antosik P, Brüssow KP, Bruska M, Nowicki M, Zabel M, Kempisty B. Amino acids metabolism and degradation is regulated during porcine oviductal epithelial cells (OECs) primary culture in vitro - a signaling pathways activation approach. Med J Cell Biol. 2018;6:18-26; DOI:10.2478/ acb-2018-0004

9. Kulus M, Józkowiak M, Kulus J, Popis M, Borowiec B, Stefańska K, Celichowski P, Nawrocki MJ, Bukowska D, Brüssow KP, Kempisty B, Jeseta M, Antosik P. "Cell cycle process", "cell division" and "cell proliferation" belong to ontology groups highly regulated during long-term culture of porcine oviductal epithelial cells. Med J Cell Biol. 2019;7:15-24; DOI:10.2478/acb-2019-0003.

10. Stefańska K, Chamier-Gliszczyńska A, Jankowski M, Celichowski P, Kulus M, Rojewska M, Antosik P, Bukowska D, Bruska M, Nowicki M, Kempisty B, Jeseta M, Zakova J. Epithelium morphogenesis and oviduct development are regulated by significant increase of expression of genes after long-term in vitro primary culture - a microarray assays. Med J Cell Biol. 2018;6:195-204; DOI:10.2478/acb-2018-0030.

11. Budna J, Celichowski P, Knap S, Jankowski M, Magas M, Nawrocki MJ, Ramlau P, Nowicki A, Rojewska M, Chermuła B, Jeseta M, Antosik P, Bukowska D, Bruska M, Zabel M, Nowicki M, Kempisty B. Fatty Acids Related Genes Expression Undergo Substantial Changes in Porcine Oviductal Epithelial Cells During Long-Term Primary Culture. Med J Cell Biol. 2018;6:39-47; DOI:10.2478/acb-2018-0008.

12. Huang DW, Sherman BT, Tan Q, Kir J, Liu D, Bryant D, Guo Y, Stephens R, Baseler MW, Lane HC, Lempicki RA. DAVID Bioinformatics Resources: expanded annotation database and novel algorithms to better extract biology from large gene lists. Nucleic Acids Res. 2007;35:W169-75; DOI:10.1093/nar/gkm415.

13. Walter W, Sánchez-Cabo F, Ricote M. GOplot: an R package for visually combining expression data with functional analysis: Fig. 1. Bioinformatics. 2015;31:2912-4; DOI:10.1093/bioinformatics/btv300.

14. von Mering C, Jensen LJ, Snel B, Hooper SD, Krupp M, Foglierini M, Jouffre N, Huynen MA, Bork P. STRING: known and predicted protein-protein associations, integrated and transferred across organisms. Nucleic Acids Res. 2005;33:D433-7; D0I:10.1093/nar/gki005.

15. Krupenko NI, Dubard ME, Strickland KC, Moxley KM, Oleinik N V., Krupenko SA. ALDH1L2 Is the Mitochondrial Homolog of 10-Formyltetrahydrofolate Dehydrogenase. J Biol Chem. 2010;285:23056; DOI:10.1074/ JBC.M110.128843.

16. Strickland KC, Krupenko NI, Dubard ME, Hu CJ, Tsybovsky Y, Krupenko SA. Enzymatic Properties Of Aldh112, A Mitochondrial 10-Formyltetrahydrofolate Dehydrogenase. Chem Biol Interact. 2011;191:129; DOI:10.1016/J.CBI.2011.01.008.

17. Sluyter R. The P2X7 Receptor. Adv. Exp. Med. Biol., vol. 1051, 2017, p. 17-53; DOI:10.1007/5584_2017_59.

18. Di Virgilio F. P2RX7: A receptor with a split personality in inflammation and cancer. Mol Cell Oncol. 2016;3:e1010937; DOI:10.1080/23723556 2015.1010937.

19. Zhou B, Westaway SK, Levinson B, Johnson MA, Gitschier J, Hayflick SJ. A novel pantothenate kinase gene (PANK2) is defective in HallervordenSpatz syndrome. Nat Genet. 2001;28:345-9; DOI:10.1038/ng572.

20. Leonardi R, Zhang Y, Rock C, Jackowski S. Coenzyme A: Back in action. Prog Lipid Res. 2005;44:125-53; DOI:10.1016/j.plipres.2005.04.001.

21. Leonardi R, Rehg JE, Rock CO, Jackowski S. Pantothenate kinase 1 is required to support the metabolic transition from the fed to the fasted state. PLoS One. 2010;5:e11107; DOI:10.1371/journal.pone.0011107.

22. Watkins PA, Maiguel D, Jia Z, Pevsner J. Evidence for 26 distinct acyl-coenzyme A synthetase genes in the human genome. J Lipid Res. 2007;48:2736-50; DOI:10.1194/jlr.M700378-JLR200.

23. Paton CM, Ntambi JM. Biochemical and physiological function of stearoyl-CoA desaturase. Am J Physiol Endocrinol Metab. 2009;297:E2837; DOI:10.1152/ajpendo.90897.2008. 
24. Koeberle A, Löser K, Thürmer M. Stearoyl-CoA desaturase-1 and adaptive stress signaling. Biochim Biophys Acta - Mol Cell Biol Lipids. 2016;1861:1719-26; DOI:10.1016/j.bbalip.2016.08.009.

25. Tondo M, Calpena E, Arriola G, Sanz P, Martorell L, Ormazabal A, Castejon E, Palacin M, Ugarte M, Espinos C, Perez B, Perez-Dueñas B, Pérez-Cerda C, Artuch R. Clinical, biochemical, molecular and therapeutic aspects of 2 new cases of 2-aminoadipic semialdehyde synthase deficiency. Mol Genet Metab. 2013;110:231-6; DOI:10.1016/J.YMGME.2013.06.021

26. Sacksteder KA, Biery BJ, Morrell JC, Goodman BK, Geisbrecht B V, Cox RP, Gould SJ, Geraghty MT. Identification of the alpha-aminoadipic semialdehyde synthase gene, which is defective in familial hyperlysinemia. Am J Hum Genet. 2000;66:1736-43; DOI:10.1086/302919.

27. Cui L, Cheng Z, Liu Y, Dai Y, Pang Y, Jiao Y, Ke X, Cui W, Zhang Q Shi J,

Fu L. Overexpression of PDK2 and PDK3 reflects poor prognosis in acute myeloid leukemia. Cancer Gene Ther. 2018; DOI:10.1038/ s41417-018-0071-9.

28. Korotchkina LG, Patel MS. Site specificity of four pyruvate dehydrogenase kinase isoenzymes toward the three phosphorylation sites of human pyruvate dehydrogenase. J Biol Chem. 2001;276:37223-9; DOI:10.1074/jbc.M103069200.

29. Dahiya R, Mohammad T, Roy S, Anwar S, Gupta P, Haque A, Khan P, Kazim SN, Islam A, Ahmad F, Hassan MI. Investigation of inhibitory potential of quercetin to the pyruvate dehydrogenase kinase 3: Towards implications in anticancer therapy. Int J Biol Macromol. 2019;136:1076-85; DOI:10.1016/j.ijbiomac.2019.06.158.

30. Doll S, Proneth B, Tyurina YY, Panzilius E, Kobayashi S, Ingold I, Irmler M, Beckers J, Aichler M, Walch A, Prokisch H, Trümbach D, Mao G, Qu F, Bayir H, Füllekrug J, Scheel CH, Wurst W, Schick JA, Kagan VE, Angeli JPF, Conrad M. ACSL4 dictates ferroptosis sensitivity by shaping cellular lipid composition. Nat Chem Biol. 2017;13:91-8; DOI:10.1038/ nchembio.2239.

31. Yuan H, Li X, Zhang X, Kang R, Tang D. Identification of ACSL4 as a biomarker and contributor of ferroptosis. Biochem Biophys Res Commun 2016;478:1338-43; DOI:10.1016/j.bbrc.2016.08.124.

32. Huang Y-W, Luo J, Weng Y-I, Mutch DG, Goodfellow PJ, Miller DS, Huang TH-M. Promoter hypermethylation of CIDEA, HAAO and RXFP3 associated with microsatellite instability in endometrial carcinomas. Gynecol Oncol. 2010;117:239-47; DOI:10.1016/j.ygyno.2010.02.006.

33. Bakkum-Gamez JN, Wentzensen N, Maurer MJ, Hawthorne KM, Voss JS, Kroneman TN, Famuyide AO, Clayton AC, Halling KC, Kerr SE, Cliby WA, Dowdy SC, Kipp BR, Mariani A, Oberg AL, Podratz KC, Shridhar V, Sherman ME. Detection of endometrial cancer via molecular analysis of DNA collected with vaginal tampons. Gynecol Oncol. 2015;137:14-22; DOI:10.1016/j.ygyno.2015.01.552.

34. Nawrocki MJ, Celichowski P, Budna J, Bryja A, Kranc W, Ciesiółka S, Borys S, Knap S, Jeseta M, Khozmi R, Bukowska D, Antosik P, Brüssow KP, Bruska M, Nowicki M, Zabel M, Kempisty B. The blood vessels development, morphogenesis and blood circulation are three ontologic groups highly up-regulated in porcine oocytes before in vitro maturation. Adv Cell Biol. 2017;5; DOI:10.1515/acb-2017-0012. 